\title{
Construction of Laboratory Teaching and Management Platform Based on Desktop Cloud
}

\author{
Yang Sheng ${ }^{*}$ 1st (Author) \\ School of Management \\ Wuhan University of Science and Technology \\ Wuhan, China \\ e-mail: shengyang@wust.edu.cn
}

\author{
Ruijun Zhang 2nd (Author) \\ School of Management \\ Wuhan University of Science and Technology \\ Wuhan, China \\ e-mail: zrjdoctor@126.com
}

\author{
Jinglian Xie 3rd (Author) \\ School of Management \\ Wuhan University of Science and Technology \\ Wuhan, China \\ e-mail: xiejinglian@wust.edu.cn
}

\begin{abstract}
In order to improve students' practical skills and innovative abilities, higher education must focus on developing and managing practical teaching. Targeting at the problems existing in the practical teaching management platform under the traditional management mode, this article presents a fullfeatured laboratory teaching and management platform based on desktop cloud, which realizes the functions of laboratory reservation, students' attendance management, network and equipment management, course resource management and practical teaching information database. In addition, the support for mobile terminals [mobile phones, laptops, table ts, etc.] on this platform has enabled the sharing of the laboratory information, improved the openness of laboratories and facilitated teaching and learning. The evaluation demonstrates that the cloud-based teaching and management platform has functioned well in the School of Management of WUST and improved the quality of practical teaching, thus providing reference for the construction of laboratory teaching and management platform in the cloud era.
\end{abstract}

Keywords-desktop cloud; practical teaching; management platform; laboratory construction

\section{INTRODUCTION}

With the advent of the information age, modern educational technology is gaining wider application in the field of higher education. Nowadays, theory lecturing and practical teaching depend heavily on computer technology, especially the latter, which has high demands on information management technology in laboratory. The cultivation of innovative talents cannot be achieved without improving students' practical skills on one hand and advanced laboratory management platform on the other. However, the current construction and management of laboratories still remain problematic, thus hindering the development of practical teaching and affecting the quality of practical teaching. How to build a smart, efficient and integrated information-based laboratory teaching and

Supported by the Key Program of Education Sciences Planning of Hubei Province of China (Grant No. 2017GA015) and by Higher Education Research Program of Hubei Province of China (Grant No. 2016238) management platform has become a pressing issue to be resolved for many colleges and universities.

Cloud computing is a state-of-the art information technology that has been widely used and generalized. As a typical application of cloud computing, desktop cloud can use virtualized applications to distribute unified teaching resources stored in cloud resource pool to various operating terminals, providing services via virtual desktops. Compared with conventional laboratory solutions, cloud-based management model has obvious advantages, such as stability, flexibility, lower cost, more efficient use of computing equipment, improved application software availability and so on. [1] Those benefits can remedy the existing deficiencies of traditional laboratory management mode. Therefore the advent of cloud computing indicates a new path for laboratory construction.

\section{PRESENT DISADVANTAGES OF CONVENTIONAL LABORATORY} TEACHING AND MANAGEMENT PLATFORM

In recent years, colleges and universities have been working to improve practical teaching environment and strengthen the construction of laboratory teaching and management platform, contributing to a higher level of practical teaching. However, some university laboratories still adopt the traditional management mode. The disadvantages in traditional management mode are as follows.

\section{A. Lack of Flexibility}

Conventional practical teaching management mode fails to meet the demands of cultivating students' creativity and practical skills and enhancing students' comprehensive qualities. Under conventional teaching management mode, the absence of laboratory information system plus the outdated network technology and many other factors lead to the limitation of opening time and space of laboratory and contents of the practical curriculum. Students cannot select the practical courses they are interested in or initiate research projects using lab resources just by themselves. Furthermore most of 
laboratory resources are inaccessible after regular school hours. Thus the traditional laboratory management model no longer satisfies the requirement of fully open labs.

\section{B. Lack of Trace Management System}

At present many laboratories still adopt manual management mode. The laboratory staff uses paper archives to record schedules of practical courses, progresses of daily experiments and class performances. However, course tutors cannot know every student's duration or the progress of experiments through paper archives. Due to the lack of educational data tracing, it is difficult for course tutors to get students' accurate feedback. Therefore, the training programs may not be suitable for the students. As for laboratory staff, they cannot obtain the precise information of experiments' duration and the use of laboratory equipment and software, resulting in certain errors in laboratory annual report. Once analyzing the annual report, laboratory staffs are unable to find strong data to support decisions on laboratory management.

\section{Lack of Effective Monitoring of Laboratory Equipment}

With the growing importance of practical teaching in higher education, more and more practical courses appear in students' training programs and the amount of laboratory equipment increases sharply. Under traditional manual management mode, problems would easily arise as follows: inconvenience of searching and registering equipment; ambiguity of current equipment status (in use, out of service and obsolete); no tracing system for equipment damage. In the event of a network or equipment failure, laboratory staff needs to spend plenty of time troubleshooting, hence affecting the quality of practical teaching.

\section{Overview of Desktop Cloud Technology}

The definition of cloud computing proposed by the National Institute of Standards and Technology (NIST) in the US emphasizes the standard features widely used in the cloud computing community:

"Cloud computing is a model for enabling convenient, ondemand network access to a shared pool of configurable computing resources (e.g., networks, servers, storage, applications, and services) that can be rapidly provisioned and released with minimal management effort or service provider interaction.”[2]

According to the service model, cloud computing can be divided into three basic models: Infrastructure as a Service (IaaS), Platform as a Service (PaaS), and Software as a Service (SaaS).[3] The three deployment models of could computing are private cloud, public cloud and hybrid cloud.[4]

Desktop cloud (or desktop virtualization), which combines the PaaS and SaaS, is one of the most significant application areas of cloud computing and also one of the most characteristic applications. Through thin clients or any other network-connected terminals, desktop cloud allows users to access the personal virtualized desktop stored in the servers and various applications in the system with the help of the dedicated client program or browsers. Thin clients installed the standalone embedded operating system use remote desktop protocol (RDP) to access the virtualized desktop in the servers.
All the resource consumption of operating systems and software happens on the cloud servers so that thin clients do not perform the tasks of calculation and storage, but providing the human-computer interaction.[5] Nowadays there are two main technical architecture of desktop cloud: Virtual Desktop Infrastructure(VDI) and Virtual OS Infrastructure(VOI). [6]

\section{Advantages of Cloud-BAsed Laboratory Teaching AND MANAGEMENT PlatForm}

The cloud-based teaching and management platform shows the advantages as follows:

\section{A. Saving Purchase Cost}

Due to the desktop virtualization, the cloud users adopt the thin clients instead of conventional PCs. The cost of a thin client is much lower than that of a conventional PC. For instance, a conventional PC's price is about 5000 yean. However, the same grade of a thin client's price is about 3000 yean (including the monitor). As for the update cycle, the PC's average update cycle is typically $3 \sim 5$ years, while the thin client can be updated every ten years. If the processing capability of virtualized desktop needs to be improved, the only thing that laboratory staff should do is to upgrade the servers' CPUs or expand the memory of servers without replacing the existing thin client with a new one.

\section{B. Reducing maintenance cost}

The hardware requirements of a thin client are minimal compared to that of a conventional PC. Thin clients use integrated system structure which contributes to their stable performance and low breakdown rate. The primary role of a thin client is to access the cloud, display the server-side data and deliver commands of input devices to the servers. All the calculation and data storage take place in the servers. Therefore the data backup, operating system installation and safety management are all conducted on the serve side. The laboratory staff's work is to maintain the servers, saving a lot of effort and cost to manage the lab resources.

\section{Sustainable energy conservation}

The energy consumption of the majority of conventional computers is above $200 \mathrm{w}$, while that of a thin client is about 20w. The mode that combines the thin clients and the cloud servers can reduce the energy consumption by $90 \%$ approximately. It leads to a significant decrease in power consumption and carbon dioxide emissions, thus creating an environment-friendly laboratory. [7]

\section{Fully open laboratory}

Since all the operating systems and application software reside on the cloud servers, teachers and students can access the virtualized desktops not only in laboratory but also from anywhere via internet-connected terminals. Thus the students' self-learning and teachers' telecommuting can be achieved. 


\section{CONSTRUCTION OF CLOUD-BASED LABORATORY TEACHING} AND MANAGEMENT PLATFORM

\section{A. Design of the Platform}

The Laboratory Teaching and Management Platform Based on Desktop Cloud consists of laboratory reservation management module (such as course release, course selection and reservation approval); practical course attendance management module (such as student login, practical course attendance and query of practical course records); network and equipment management module (such as condition monitoring of network and equipment and fault alarm); course resource management module (such as digital textbook, electronic courseware, online course, exercise and exam); database of practical teaching information (such as laboratory annual report, trace management). Meanwhile, the platform supports various mobile devices (phones, laptops, tablets, etc.), bringing great convenience to lab users. The framework of this platform's overall functions is shown in Fig.1.

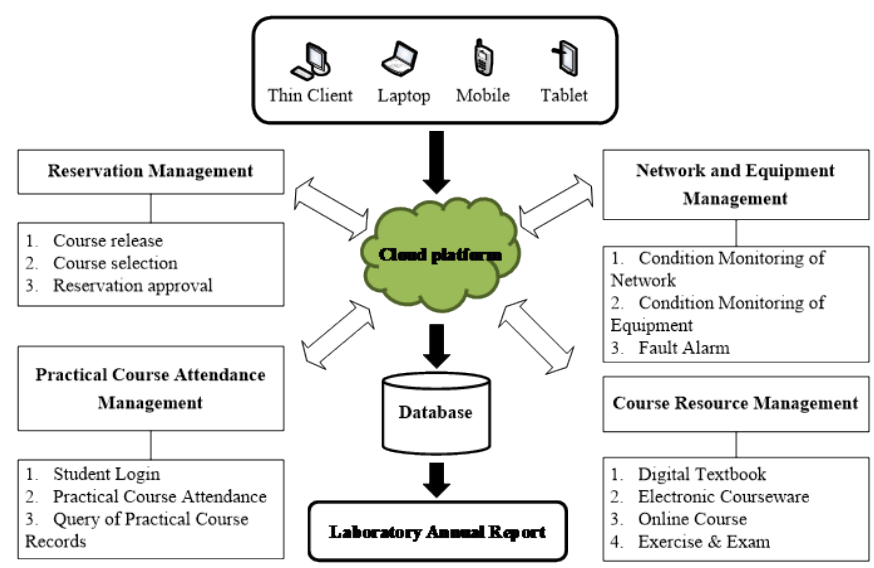

Fig. 1. The framework of the laboratory teaching and management platform platform's overall functions

\section{1) Laboratory Reservation Management Module}

The module is composed of three parts: course release, course selection and reservation approval. The platform allows more flexible reservation procedure. Firstly the tutors post the syllabus and time of practical courses according to the training programs. Then the students select the suitable course time based on their own time schedules and reserve the lab equipment. Finally the laboratory staff approves the students' reservations.

\section{2) Practical Course Attendance Management Module}

The module includes student login, practical course attendance and query of practical course records. When students log in the lab platform and access the software using the thin client in the lab or their own mobile devices, if their reservations exist, the attendance management system would record the durations of experiments and the software they use.

\section{3) Network and Equipment Management Module}

The module includes condition monitoring of network and equipment, fault early warning and fault alarm. By using cloud platform and the network and equipment management system, an intelligent integrated management platform can be built to monitor the running state of lab's network, severs, virtual machine, applications and operating system around the clock. This platform provides a rapid and straightforward way for laboratory staff to monitor all the lab's resources. If malfunctions occur, the system will provide the fault alarms and indicate the point of failure to the lab staff timely by means of sending e-mails or messages.

\section{4) Course Resource Management Module}

In this module, an e-learning platform is realized which is primarily used for tutors to post the course resources online such as digital textbooks, electronic courseware, online courses, exercises and exams, etc. On line, the students sign up the courses they like for self-study; off line, the tutors then elaborate the key points and explain the difficulties in class. Meanwhile the tutors can also give self-study assignments to students after class. This module realizes the "flipped classroom".

\section{5) Database of Practical Teaching Information}

Taking advantage of big data of cloud computing, useful data can be extracted from the lab system to generate an annual report that our school requires. This data includes the students' duration of using lab computers, lab software and equipment they use, the progress of experiments and so on. The statistical analysis of practical teaching information provides necessary and enough support for the teaching management and further educational reform.

\section{6) Remote Laboratory}

Using the desktop cloud technology, the application services are allowed to be accessed by the browsers wherever they are. Students can use their own computers, mobile phones, tablets and other mobile terminals to use the software in laboratory. The remote laboratory realizes the information sharing and improves the openness of laboratory.

\section{B. Use of the Platform}

The entire cloud storage and computational resource are controlled by the laboratory of the School of Management, not by the public cloud service providers. Physical isolation ensures the safety of data. The topological structure of this teaching and management platform is shown in Fig. 2.

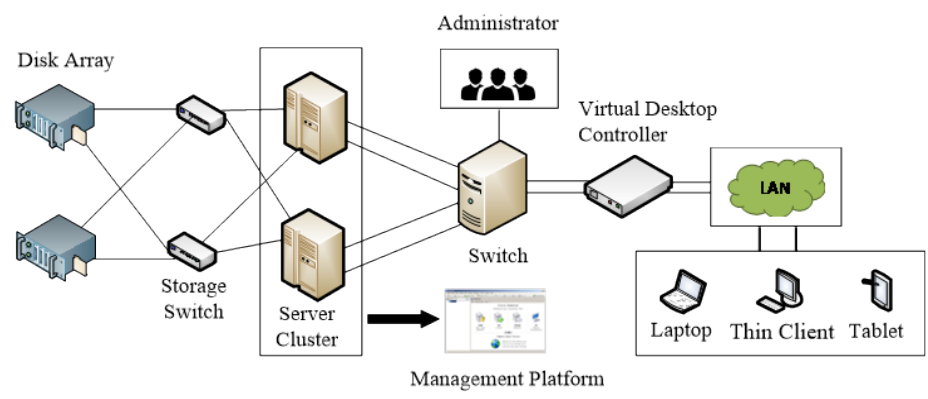

Fig. 2. The topological structure of the laboratory teaching and management platform 
Now the cloud-based laboratory teaching and management platform of School of Management mainly performs the practical teaching task of about 50 practical courses from 10 undergraduate majors. Above 150 software applications are installed in the platform such as BIM software, ERP software, virtual simulation software, Java software, accounting software, logistics management software. In addition, students can work on their graduation theses and graduation projects, and professors can conduct research projects by using the platform. The cloud-based teaching and management platform has functioned well and produced remarkable results in our school.

\section{1) The process of practical teaching is optimized}

Using the data mining of practical teaching big data, the tutors can learn about the student's major difficulties of practical courses and explain those difficulties in details, thus optimizing the process of practical teaching.

\section{2) The laboratory resource is fully utilized}

The laboratory reservation function is conducive to higher efficiency of the course reservation and approval process. The space and equipment of laboratory is fully and scientifically utilized.

\section{3) The management process of practical courses is optimized}

The teaching and management platform monitors the network and equipment of lab. The laboratory staff can be informed of the fault information as soon as the breakdown occurs, which shortens the repair time and ensure that the practical courses are conducted normally.

\section{CONCLUSION}

This paper presents a laboratory teaching and management platform based on desktop cloud which improves the openness of laboratory, meets the tutors' and students' demands of accessing teaching resources anytime and anywhere, and realizes the students' self-study, teachers' telecommuting and the "flipped classroom". The platform optimizes the process of practical teaching and the management of practical courses, also saves the labor cost and resource consumption, and significantly improve the efficiency of practical teaching.

\section{REFERENCES}

[1] A.C. Caminero, S. Ros, R. Hernandez, A. Roblesgomez, L. Tobarra and P.J. Tolbanosgranjo, "Virtual remote laboratories management System (TUTORES): Using cloud computing to acquire university practical skills,” IEEE Transactions on Learning Technologies, Vol.9, pp. 133145, June 2016.

[2] P. Mell and T. Grance, "SP 800-145. The NIST Definition of Cloud Computing,” National Institute of Standards \& Technology, 2011.

[3] J. Paredesgualtor, O. Moscosozea, P. Saa, F. Sandoval, P. Rodas, "Unified cloud computing adoption framework," 2017 International Conference on Information Systems and Computer Science (INCISCOS), 2017, pp. 247-252.

[4] D.L. Chen, M. Fu, L. Chen, "Study on construction mode of experimental teaching platform in colleges and universities based on hybrid cloud," Experimental Technology and Management, Vol.30, pp.63-66, May 2013. (In Chinese)

[5] F. Qin, S.B. Huang, W.L. Qin, A.P. Liao, "Research on management of multimedia computer laboratory based on desktop clouds," Experimental Technology and Management, Vol.32, pp.164-166, March 2015. (In Chinese)

[6] J.G Huang, "Research and implementation of desktop cloud architecture in college teaching environment," Computer Technology and Development, Vol.23, pp.222-225, December 2013. (In Chinese)

[7] B. Jin, X.J. Zheng, Z.Q. Yao, J. Lin "Desktop virtualization and management of computer laboratory," Experimental Technology and Management, Vol.31, pp.85-88, February 2014. (In Chinese) 